\title{
Brief Reports of Nobel Laureates in Physics
}

\author{
Dr.Shaikh Sarfaraz Ali \\ Department of Physics, Veer Surendra Sai University of Technology (VSSUT), Burla-768018, Samalpur, Odisha, \\ India.
}

\begin{abstract}
Alfred B. Nobel, a Swedish chemist and engineer who invented dynamite left \$9 million in his will to establish the Nobel Prize, which are awarded annually, without regard to nationality, in six different areas like Peace, Literature, Physics, Chemistry, Physiology or Medicine and Economic Science to those who, during the preceding year, shall have conferred the greatest benefit on mankind. Here the complete list of all the Nobel Laureates in Physics since 1901 to 2013 is compiled.
\end{abstract}

1901-Wilhelm Conrad Rontgen, Born in Lennep, Rhenish Prussia, Germany (1845-1923) was awarded the Nobel Prize for the Discovery of Rontgen rays which is also known as X-rays.

1902-Hendrik Antoon Lorentz, Born in Arnhen, the Netherlands (1853-1928) was awarded 1/2 of the Nobel Prize for the investigations of effects of magnetism on the phenomena of radiation.

1902-Pieter Zeeman, Born in Zonnemaire, the Netherlands (1865-1943) was awarded 1/2 of the Nobel Prize for the Investigations of the effects of magnetism on the phenomena of radiation.

1903-Henri Antoine Becquerel, Born in Paris (1852-1908) was awarded 1/2 of the Nobel Prize for the discovery of spontaneous radioactivity.

1903-Pierre Curie, Born in Paris (1859-1906) was awarded 1/4 of the Nobel Prize for the Phenomena of radiation discovered by Becquerel.

1903-Marie Sklodovska Curie, Born in Warsaw, France (1867-1934) was awarded 1/4 of the Nobel Prize for the Phenomena of radiation discovered by Becquerel.

1904-Lord Rayleigh (John William Strutt), Born in Essex, England (1842-1919) was awarded the Nobel Prize for the Investigations on the densities of most important gases, and for the discovery of argon, one of the results of those investigations.

1905-Philipp Eduard Anton Von Lenard, Born in Pressburg, then Austria-Hungary (1862-1947) was awarded the Nobel Prize for the work on cathode rays.

1906-Joseph John Thomson, Born in Cheetham Hall, Near Manchester, United Kingdom (1856-1940) was awarded the Nobel Prize for the Theoretical and experimental investigations on passage of electricity through gases.

1907-Albert Abraham Michelson, Born in Streino, Prussia, Germany (1852-1931) was awarded the Nobel Prize for the optical instruments of precision and the spectroscopic and meteorological investigations which he carried out by means of them.

1908-Gabriel Lippmann, Born in Hollerich, Luxemburg, France (1845-1921) was awarded the Nobel Prize for the method of photographic reproduction of colors, based upon the phenomenon of interferences.

1909-Karl-Ferdinand Braun, Born in Fulda, Germany (1850-1918) was awarded 1/2 of the Nobel Prize for the development of wireless telegraphy.

1909-Guglielmo Marconi, Born in Bologana, Italy (1874-1937) was awarded 1/2 of the Nobel Prize for the development of wireless telegraphy.

1910-Johannes Diderik Van der Waals, Born in Leyden, the Netherlands (1837-1923) was awarded the Nobel Prize for the work on equation of state of gases and liquids.

1911-Wilhelm Wien, Born in Geffken, East Prussia, Germany (1864-1928), was awarded the Nobel Prize for the discoveries regarding the laws governing the radiation of heat.

1912-Nils Gustaf Dalen, Born in Stenstorp, Sweden (1869-1937) was awarded the Nobel Prize for the invention of automatic regulators that can be used in conjunction with gas accumulators for lighting the lighthouses and light buoys .

1913-Heike Kamerlingh Onnes, Born in Groningen, the Netherlands (1853-1926) was awarded the Nobel Prize for the Investigations on the properties of matter at low temperatures, which led inter-alia to the production of liquid helium.

1914-Max Von Laue, Born in Phaffendorf on the Rhine, Germany (1879-1960) was awarded the Nobel Prize for the Discovery of diffraction of Rontgen rays (x-rays) in crystals.

1915-Sir William Henry Bragg, Born in Westward, Cumberland, United Kingdom (1862-1942) was awarded 1/2 of the Nobel Prize for the analysis of crystal structure by means of X-rays.

1915-William Lawrence Bragg, S/o Sir William Henry Bragg, Born in Adelaide, South Australia (1890-1971) 
was awarded $1 / 2$ of the Nobel Prize for the study of crystal structure by means of X-rays.

1916-The Nobel Prize money was allocated to the special fund of this Prize section.

1917-Charles Glover Barkla, Born in Widnes, Lancashire, United Kingdom (1877-1944) was awarded the Nobel Prize for the discovery of the characteristic Rontgen radiation of the element.

1917-Henry G. J. Moseley, Born in Weymouth, on England's south coast (1887-1915). From the data he was able to infer a relationship between the x-ray wavelength of an element and its atomic number, a relationship that permitted him to correct ambiguities in then-current atomic number assignments and to predict the existence of several then-unknown elements. He would have also shared the Nobel Prize with Charles Glover Bakla, but for his death at Galipoli.

1918-Max Karl Ernst Ludwig Planck, Born in Kiel, Germany (1858-1947) was awarded the Nobel Prize for the development of Physics by the discovery of energy quanta.

1919-Johannes Stark, Born in Schickenhof, Bavaria, Germany (1874-1957) was awarded the Nobel Prize for the discovery of the Doppler effect in canal rays and the separation of spectral lines in an electric fields.

1920-Charles Edouard Guillaume, Born in Fleuriar, Switzerland (1861-1938) was awarded the Nobel Prize for the Precise measurements in Physics by the discovery of anomalies in nickel-steel alloys.

1921-Albert Einstein, Born in Ulm, Wurtemberg, Germany (1879-1955) was awarded the Nobel Prize for the attainments in mathematical physics, theory of Relativity and especially for the discovery of law of the photo electric effect.

1922-Neils Henrik David Bohr, Born in Copenhagen, Denmark (1885-1962) was awarded the Nobel Prize for the Investigation of the structure of atoms and of the radiation emanating from them.

1923-Robert Andrews Millikan, Born at Morrison, Illinois, USA (1868-1953) was awarded the Nobel Prize for the work on elementary charge of electricity and also on photoelectric effect.

1924-Karl Manne Georg Siegbahn, Born in Orebro, Sweden (1886-1978) was awarded the Nobel Prize for the Research and discoveries in X-ray (measurements of X-ray wavelength) spectroscopy.

1925-James Franck, Born in Hamburg, Germany (1882-1964) was awarded 1/2 of the Nobel Prize for the discovery of the laws governing the impact of an electron upon an atom.

1925-Gustav Ludwig Hertz, Born in Hamburg, Germany (1887-1975) was awarded 1/2 of the Nobel Prize for the Discovery of the laws governing the impact of an electron upon an atom.

1926-Jean Baptiste Perrin, Born in Lille, France (1870-1942) was awarded the Nobel Prize for the work on the discontinuity in the structure of matter, and in particular for the discovery of the equilibrium of sedimentation.

1927-Charles Thomson Rees Wilson, Born in Glencorse, Near Edinburg, United Kingdom (1869-1959) was awarded 1/2 of the Nobel Prize for the Discovery of a method of rendering discernible the paths of electrically charged particles by the condensation of vapor.

1927-Arthur Holly Compton, Born in Wooster, Ohio, USA (1892-1962) was awarded 1/2 of the Nobel Prize for the Discovery of Compton effect, which confirmed the quantum theory of radiation and assigned mass as well as energy to light quanta.

1928-Sir Owen Willians Richardson, Born in Dewsbury, Yorkshire, United Kingdom (1879-1959) was awarded the Nobel Prize for the work on the thermionic phenomena and especially for discovering the law which bears his name.

1929-Prince Louis (Victor Pierre Raymond de Broglie), Born at Dieppe, France (1892-1987) was awarded the Nobel Prize for the discovery of wave character of electrons.

1930-Sir Chandrasekhar Venkata Raman, Born in Trichinopoly,Tamilnadu, India (1888-1970) was awarded the Nobel Prize for the Discovery of scattering of light which is well known as Raman Effect: that the radiation is scattered by various substances with a change in frequency, the change being characteristic of the scattering atoms or molecules. Why the sky looks blue and why the sea water looks blue?

1931-The Nobel Prize money was allocated to the special fund of this section.

1932-Werner Heisenberg, Born in Duisberg, Rhenish Prussia, Germany (1901-1976) was awarded the Nobel Prize for the Creation of quantum mechanics (matrix mechanics), whose application has led to the discovery of allotropic forms of hydrogen.

1933-Paul Adrien Maurice Dirac, Born in Bristol, United Kingdom (1902-1982) was awarded 1/2 of the Nobel Prize for the Creation of wave mechanics, mathematical theory of quantum mechanics: statistics.

1933-Erwin Schrodinger, Born in Wien, Austria (1887-1961) was awarded 1/2 of the Nobel Prize for the creation of wave mechanics, a theory of Interactions of elementary particles.

1934-The Nobel Prize money was with $1 / 3$ allocated to the main fund and with $2 / 3$ to the special fund of this prize section.

1935-Sir James Chadwick, Born in Manchester, United Kingdom (1891-1974) was awarded the Nobel Prize for the discovery of the neutron.

1936-Victor Franz Hess, Born in Waldstein castle, Near Peggau, Austria (1883-1964) was awarded 1/2 of the Nobel Prize for the Discovery of cosmic radiation. 
1936-Carl David Anderson, Born in NewYork City (1905-1991) was awarded 1/2 of the Nobel Prize for the discovery of the Positron (anti-electron).

1937-Clinton Joseph Davisson, Born in Bloomington, Illinois,USA (1881-1958) was awarded 1/2 of the Nobel Prize for the Experimental discovery of the diffraction of electrons by crystals.

1937-Sir George Paget Thomson, Born in Cambridge, England (1892-1975) was awarded 1/2 of the Nobel Prize for the Experimental discovery of the diffraction of electrons by crystals.

1938-Enrico Fermi, Born in Rome, Italy (1901-1954) was awarded the Nobel Prize for the Identification of new radioactive elements produced by Neutron bombardment and the discovery of nuclear reactions affected by slow neutrons.

1939-Ernest Orlando Lawrence, Born in Canton, South Dakota, USA (1901-1958) was awarded the Nobel Prize for the Invention and development of the cyclotron and for the production of artificial radioactive elements.

1940-The Nobel Prize money was with $1 / 3$ allocated to the main fund and with $2 / 3$ to the special fund of this prize section.

1941-The Nobel Prize money was with $1 / 3$ allocated to the main fund and with $2 / 3$ to the special fund of this prize section.

1942-The Nobel Prize money was with $1 / 3$ allocated to the main fund and with $2 / 3$ to the special fund of this prize section.

1943-Otto Stern, Born in Sohrau, Germany (1888-1969) was awarded the Nobel Prize for the Contribution to the development of the molecular-ray method of detecting the magnetic moment of proton.

1944-Isidor Isaac Rabi, Born in Rymanow, then Austria-Hungary (1898-1988) was awarded the Nobel Prize for the Extraordinary research in the resonance method of recording the magnetic properties of nuclei.

1945-Wolfgang Pauli, Born in Wien, Austria (1900-1958) was awarded the Nobel Prize for the discovery of the exclusion principle, which deals with regulation of electrons in the outer shell of atoms, which is also called Pauli Exclusion Principle.

1946-Percy Williams Bridgeman, Born in Cambridge, Massachusetts, USA (1882-1961) was awarded the Nobel Prize for the Investigation of an apparatus to produce extremely high pressure, and for the discoveries he made therewith in the field of high pressure Physics.

1947-Sir Edward Victor Appleton, Born in Bradford, Yorkshire, United Kingdom (1892-1965) was awarded the Nobel Prize for the extraordinary research in physics of the upper atmosphere, particularly for the discovery of ionized layer called as Appleton layer.

1948-Patrick Maynard Stuart Blackett, Born in London (1897-1974) was awarded the Nobel Prize for the development of Wilson cloud chamber method and discoveries therewith in the field of nuclear physics and cosmic radiation.

1949-Hideki Yukawa, Born in Tokyo (1907-1981) was awarded the Nobel Prize for the Prediction of the existence of mesons, based upon the theory of nuclear forces.

1950-Cecil Frank Powell, Born in Tonbridge, Kent,United Kingdom (1903-1969) was awarded the Nobel Prize for the Development of a simple method of examining the action of nuclei by the photography (Nuclear Emulsion Technique) and also for important discoveries concerning the production of $\pi$ Mesons which was predicted by Hideki Yukawa, Japanese Physicist.

1951-Sir John D. Cockcroft, Born in Todmorden, United Kingdom (1897-1967) was awarded 1/2 of the Nobel Prize for the discovery of transmutations of nuclei by artificially accelerated particles.

1951-Ernest T.S.Walton, Born in Dungarvan, Ireland (1903-1995) was awarded 1/2 of the Nobel Prize for the production of nuclear transmutations.

1952-Edward Mills Purcell, Born in Taylorville, Illinois,USA (1912-1997) was awarded 1/2 of the Nobel Prize for the development of new methods to measure the nuclear magnetic moments and also the discoveries in connection therewith.

1952-Felix Bloch, Born in Zurich, Switzerland (1905-1983) was awarded 1/2 of the Nobel Prize for the develop -ment of new methods to measure the Nuclear magnetic moments and also the discoveries in connection therewith.

1953-Frits Frederik Zernike, Born in Amsterdam, the Netherlands (1888-1966) was awarded the Nobel Prize for the method of phase contrast and especially for the invention of phase-contrast microscope.

1954-Max Born, Born in Breslau, then Germany (1882-1970) was awarded 1/2 of the Nobel Prize for the funda -mental work in Quantum Mechanics and especially for the statistical interpretation of wave function.

1954-Walter Bothe, Born in Oranienburg, Federal Republic of Germany (1891- 1957) was awarded 1/2 of the Nobel Prize in physics for the method of coincidence and the discoveries which it had made possible.

1955-Polykarp Kusch,Born in Blankenberg, Germany (1911-1993) was awarded 1/2 of the Nobel Prize for the precise determination of magnetic moment of electron.

1955-Wills Eugene Lamb, Jr., Born in Los Angeles, USA (1913) was awarded 1/2 of the Nobel Prize for the discoveries concerning the fine structure of the hydrogen spectrum. 
1956-William Bradford Shockley, Born in London (1910-1989) was awarded 1/3 of the Nobel Prize for the discovery of semiconductors and transistor effect.

1956-Walter Houser Brattain, Born in USA (1902-1987) was awarded 1/3 of the Nobel Prize for the discovery of semiconductors and transistor effect.

1956-John Bardeen, Born in Madison, Wisconsin, USA (1908-1991) was awarded 1/3 of the Nobel Prize for the discovery of semiconductors and transistor effect.

1957-Chen Ning Yang, Born in Hofei, China (1922) was awarded 1/2 of the Nobel Prize for the overthrow of the principle of the conservation of Parity.

1957-Tsung Dao Lee, Born in China (1926) was awarded 1/2 of the Nobel Prize for the experiments which destroyed the long accepted principle of conservation of Parity.

1958-Pavel A. Cherenkov, Born in USSR (1904-1990) was awarded 1/3 of the Nobel Prize for the discovery and interpretation of the Cherenkov Effect.

1958-Il'ja M.Frank, Born in USSR (1908-1990) was awarded 1/3 of the Nobel Prize for the discovery and interpretation of the Cherenkov Effect.

1958-Igor Y.Tamm, Born in USSR (1895-1971) was awarded 1/3 of the Nobel Prize for the discovery and interpretation of the Cherenkov Effect.

1959-Owen Chamberlain, Born in San Francisco, USA (1920) was awarded 1/2 of the Nobel Prize for the discovery of the anti-proton.

1959-Emilio Gino Segre, Born in Tivoli, Italy (1905-1989) was awarded 1/2 of the Nobel Prize for the discovery of the anti-proton.

1960-Donald Arthur Glaser, Born in Cleveland, USA (1926) was awarded the Nobel Prize for the invention of the Bubble chamber.

1961-Rudolf-Ludwig Mossbauer, Born in Munich, Federal Republic of Germany (1929) was awarded 1/2 of the Nobel Prize for the resonance absorption of gamma radiation and the discovery in this connection of the effect which bears his name, Mossbauer effect.

1961-Robert Hofstadter, Born in USA (1915-1990) was awarded 1/2 of the Nobel Prize for the Pioneering studies of electron scattering in atomic nuclei and for thereby achieving discoveries concerning the structure of the nucleons.

1962-Lev Davidovich Landau, Born in Baku, USSR (1908-1968) was awarded the Nobel Prize for the Pioneering theories of condensed matter, especially for liquid helium.

1963-Eugene Paul Wigner, Born in Budapest, Hungary (1902-1995) was awarded 1/2 of the Nobel Prize for many contributions to the theory of the atomic nucleus and the elementary particles, particularly through the discovery and application of fundamental symmetry principles.

1963-Maria Goeppert Mayer, Born in Kattowitz, then Germany (1906-1972) was awarded 1/4 of the Nobel Prize for adding to man's knowledge about the nuclear shell model.

1963-J. Hans D. Jensen, Born in Federal Republic of Germany (1907-1973) was awarded 1/4 of the Nobel Prize for adding to man's knowledge about the structure of nuclear shell model.

1964-Aleksandr Mikhailovich Prokhorov, Born in USSR (1916-2002) was awarded 1/3 of the Nobel Prize for the fundamental work in the field of quantum electronics, which has led to the construction of oscillators and amplifiers based on the maser-laser principle.

1964-Charles Hard Townes, Born in Greenville, USA (1915) was awarded 1/3 of the Nobel Prize for the fundamental work in the field of quantum electronics, which has led to the construction of oscillators and amplifiers based on the maser-laser principle.

1964-Nikolai Gennadiyevich Basov, Born in USSR (1922-2001) was awarded 1/3 of the Nobel Prize for the fundamental work in the field of quantum electronics, which has led to the construction of oscillators and amplifiers based on the maser-laser principle.

1965-Richard P. Feynman ,Born in NewYork(1918-1988) was awarded 1/3 of the Nobel Prize for the fundamental work in quantum electrodynamics, with deep ploughing consequences for the physics of elementary particles.

1965-Julian Schwinger, Born in New York (1918-1994) was awarded 1/3 of the Nobel Prize for the fundamental work in quantum electrodynamics, with deep ploughing consequences for the physics of elementary particles.

1965-Sin-Itiro Tomonaga, Born in Japan (1906-1979) was awarded 1/3 of the Nobel Prize for the Fundamental work in quantum electrodynamics, with deep ploughing consequences for the physics of elementary particles.

1966-Alfred Kastler, Born in France (1902-1984) was awarded the Nobel Prize for the discovery and development of optical methods for studying the Hertzian resonance in atoms.

1967-Hans Albrecht Bethe, Born in Strasbourg, then Germany (1906) was awarded the Nobel Prize for the work on the theory of nuclear reactions, and especially for the discoveries concerning the energy production in stars. 1968-Luis Walter Alvarez, Born in San Francisco, California (1911-1988) was awarded the Nobel Prize for the 
decisive contributions to elementary Particle Physics, in particular the discovery of a large number of resonance states, made possible through his development of the technique of using hydrogen bubble chamber and data analysis.

1969-Murray Gell-Mann, Born in New York (1929), was awarded the Nobel Prize for the contributions and discoveries concerning the classification of Elementary Particles and their Interactions.

1970-Hannes Olof Gosta Alfven, Born in Sweden (1908-1995) was awarded 1/2 of the Nobel Prize for the fundamental work in magneto hydrodynamics with fruitful applications in different parts of Plasma Physics.

1970-Louis Eugene Felix Neel, Born in France (1904-2000) was awarded 1/2 of the Nobel Prize for the fundamental work and discoveries concerning Ferro- magnetism and anti-Ferro magnetism, which have led to the important applications in solid state Physics.

1971-Dannis Gabor, Born in Budapest, Hungary (1900-1979) was awarded the Nobel Prize for the Discovery and development of the holographic method.

1972-John Robert Schrieffer, Born in USA (1931) was awarded 1/3 of the Nobel Prize for the Development of theory of superconductivity, usually called as the BCS-theory.

1972-Leon Neil Cooper, Born in USA (1930) was awarded 1/3 of the Nobel Prize for the Development of theory of superconductivity, usually called as the BCS theory.

1972-John Bardeen, Born in Madison, Wisconsin, USA (1908-1999) was awarded 1/3 of the Nobel Prize for the development of theory of super conductivity, usually called the BCS-theory.

1973-Brian David Josephson, Born in United Kingdom (1940) was awarded 1/2 of the Nobel Prize for the Theoretical predictions of the properties of a super-current through a tunnel barrier, in particular those phenomena which are generally known as the Josephson effects.

1973-Ivar Giaever, Born in Bergen, Norway (1929) was awarded 1/4 of the Nobel Prize for Experimental discoveries regarding tunneling phenomena in semi-conductors and super conductors respectively.

1973-Leo Esaki, Born in Japan (1925) was awarded 1/4 of the Nobel Prize for the Experimental discoveries regarding tunneling phenomena in semi-conductors and super conductors respectively.

1974- Sir Martin Ryle, Born in United Kingdom (1918-1984) was awarded 1/2 of the Nobel Prize for the Pioneering research in the radio astrophysics, in particular for the aperture synthesis technique.

1974-Antony Hewish, Born in United Kingdom (1924) was awarded 1/2 of the Nobel Prize for the Pioneering research in the radio astrophysics, in particular his decisive role in the discovery of Pulsars.

1975-Aage Niels Bohr, Born in Denmark (1922) was awarded 1/3 of the Nobel Prize for the discovery of the connection between the collective motion and the particle motion in atomic nuclei and the development of the theory of the structure of the atomic nucleus based in this connection.

1975-Ben Roy Mottelson, Born in Chicago, USA (1926) was awarded 1/3 of the Nobel Prize for the Discovery of the connection between the collective motion and the particle motion in atomic nuclei and the development of the theory of the structure of the atomic nucleus based on this connection.

1975-Leo James Rainwater, Born in USA (1917-1986) was awarded 1/3 of the Nobel Prize for the discovery of the connection between the collective motion and the particle motion in atomic nuclei and the development of the theory of the structure of the atomic nucleus based on this connection.

1976-Burton Richter, Born in USA (1931) was awarded 1/2 of the Nobel Prize for the Pioneering work in the discovery of a heavy elementary particle of a new kind known as charm quark ( c ).

1976-Samuel Chao Chung Ting, Born in USA (1936) was awarded 1/2 of the Nobel Prize for the Pioneering work in the discovery of a heavy elementary particle of a new kind known as charm quark ( c ).

1977-Philip Warren Anderson, Born in USA (1923) was awarded 1/3 of the Nobel Prize for the Fundamental theoretical investigations of the electronic structure of magnetic and disordered system.

1977-Sir Nevill Francis Mott, Born in United Kingdom (1905-1996) was awarded 1/3 of the Nobel Prize for the fundamental theoretical investigations of the electronic structure of magnetic and disordered system.

1977-John Hasbrouck Van Vleck, Born in USA (1899-1980) was awarded 1/3 of the Nobel Prize for the fundamental theoretical investigations of the electronic structure of magnetic and disordered system.

1978-Pyotr Leonidovich Kapitsa, Born in USSR (1894-1984) was awarded 1/2 of the Nobel Prize for the Basic inventions and discoveries in the area of low-temperature Physics.

1978-Arno Allan Penzias, Born in Munich, Germany (1933) was awarded 1/4 of the Nobel Prize for the discovery of cosmic microwave background radiation.

1978-Robert Woodrow Wilson, Born in USA (1936) was awarded 1/4 of the Nobel Prize for the discovery of cosmic microwave background radiation.

1979-Sheldon Lee Glashow, Born in USA (1932) was awarded 1/3 of the Nobel Prize for the Contribution to the theory of the unified weak and electro- magnetic interaction between elementary particles, including, interalia, the prediction of the weak neutral current.

1979-Abdus Salam, Born in India (1926-1996) was awarded 1/3 of the Nobel Prize for the contribution to the theory of the unified weak and electro- magnetic interaction between elementary particles, including, inter-alia, 
the prediction of the weak neutral current.

1979-Steven Weinberg, Born in USA (1933) was awarded 1/3 of the Nobel Prize for the Contribution to the theory of the unified weak and electro- magnetic interaction between elementary particles, including, inter alia, the prediction of the weak neutral current.

1980-James Watson Cronin, Born in USA (1931) was awarded 1/2 of the Nobel Prize for the discovery of violation of fundamental symmetry principles in the decay of neutral k-mesons.

1980-Val Logsdon Fitch, Born in USA (1923) was awarded 1/2 of the Nobel Prize for the discovery of violation of fundamental symmetry principles in the decay of neutral k-mesons.

1981-Kai M. Siegbahn, Born in Sweden (1918) was awarded 1/2 of the Nobel Prize for the Contribution to the development of high resolution electron spectroscopy.

1981-Arthur Leonard Schawlow, Born in USA (1921-1999) was awarded 1/4 of the Nobel Prize for the contribution to the development of laser spectroscopy.

1981-Nicolaas Bloembergen, Born in Dordrccht, the Netherlands (1920) was awarded 1/4 of the Nobel Prize for the Contribution to the development of laser spectroscopy.

1982-Kenneth G. Wilson, Born in USA (1936) was awarded the Nobel Prize for the Theory of critical phenomena in connection with phase transitions.

1983-Subramanian Chandrasekhar, Born in Lahore, India (1910-1995) was awarded 1/2 of the Nobel Prize for the Theoretical studies of physical processes of importance to the structure and evolution of the stars.

1983-William Alfred Fowler, Born in USA (1911-1995) was awarded 1/2 of the Nobel Prize for the theoretical and experimental studies of the nuclear reactions of importance in the formation of the chemical elements in the Universe.

1984-Carlo Rubbia, Born in Italy (1934) was awarded 1/2 of the Nobel Prize for the Decisive contributions to the large project, which led to the discovery of the field particles $\mathrm{W}^{+}, \mathrm{W}^{-}$and $\mathrm{Z}^{0}$ communicators of weak interactions.

1984-Simon Van der Meer, Born in the Netherlands (1925) was awarded 1/2 of the Nobel Prize for the decisive contributions to the large project, which led to the discovery of the field Particles $\mathrm{W}^{+}, \mathrm{W}^{-}$and $\mathrm{Z}^{0}$ communicators of weak interactions.

1985-Klaus Von Klitzing, Born in Federal Republic of Germany (1943) was awarded the Nobel Prize for the discovery of the quantized Hall Effect.

1986-Ernst Ruska, Born in Federal Republic of Germany (1906-1988) was awarded 1/2 of the Nobel Prize for the Fundamental work in electron optics and for the design of the first electron microscope.

1986-Gerd Binning, Born in Federal Republic of Germany (1947) was awarded 1/4 of the Nobel Prize for design of the scanning tunneling microscope.

1986-Heinrich Rohrer, Born in Switzerland (1933) was awarded 1/4 of the Nobel Prize for the Design of the scanning tunneling microscope.

1987-Georg Bednorz, Born in Federal Republic of Germany (1950) was awarded 1/2 of the Nobel Prize for the important break-through in the discovery of superconductivity in ceramic materials.

1987-Karl Alex Muller, Born in Switzerland (1927) was awarded 1/2 of the Nobel Prize for the important breakthrough in the discovery of super-conductivity in ceramic materials.

1988-Leon M. Lederman, Born in USA (1922) was awarded 1/3 of the Nobel Prize for the Neutrino beam method and the demonstration of the doublet structure of the leptons through the discovery of the muonneutrino.

1988-Melvin Schwartz, Born in USA (1932) was awarded 1/3 of the Nobel Prize for the Neutrino beam method and the demonstration of the doublet structure of the leptons through the discovery of the muon-neutrino.

1988-Jack Steinberger, Born in Bad Kissingen, Germany (1921) was awarded 1/3 of the Nobel Prize for the Neutrino beam method and the demonstration of the doublet structure of the leptons through the discovery of the muon-neutrino.

1989-Norman F. Ramsay, Born in USA (1915) was awarded 1/2 of the Nobel Prize for the Invention of the separated oscillatory fields method and its use in the hydrogen maser and other atomic clocks.

1989-Hans. G. Dehmelt, Born in Gorlitz, Germany (1922) was awarded 1/4 of the Nobel Prize for the development of ion trap technique.

1989-Wolfgang Paul, Born in Federal Republic of Germany (1913-1990) was awarded 1/4 of the Nobel Prize for development of ion trap technique.

1990-Jerome I. Friedman, Born in USA (1930) was awarded 1/3 of the Nobel Prize for the Pioneering investigation concerning deep inelastic scattering of electrons on protons and bound neutrons, which have been of essential importance for the development of the quark model in Particle Physics.

1990-Henry W. Kendall, Born in USA (1926-1999) was awarded 1/3 of the Nobel Prize for the Pioneering investigation concerning deep inelastic scattering of electrons on protons and bound neutrons, which have been of essential importance for the development of the quark model in Particle Physics. 
1990-Richard E. Taylor, Born in Canada (1929) was awarded 1/3 of the Nobel Prize for the Pioneering investigation concerning deep inelastic scattering of electrons on protons and bound neutrons, which have been of essential importance for the development of the quark model in Particle Physics.

1991-Pierre-Gilles de Gennes, Born in France (1932) was awarded the Nobel Prize for discovering the methods developed for studying the order phenomena in simple systems can be generalized to more complex forms of matter, in particular to liquid crystals and polymers.

1992-Georges Charpak, Born in Dabrovica, Poland (1924) was awarded the Nobel Prize for the Invention and development of particle detectors, in particular the Multi-wire proportional chamber.

1993-Russell A. Hulse, Born in USA (1950) was awarded 1/2 of the Nobel Prize for the Discovery of a new type of Pulsar, which has opened up new possibilities for the study of gravitation.

1993-Josep H.Taylor Jr., Born in USA (1941) was awarded 1/2 of the Nobel Prize for the Discovery of a new type of Pulsar, which has opened up new possibilities for the study of gravitation.

1994-Bertram N. Brockhouse, Born in Canada (1918) was awarded 1/2 of the Nobel Prize for the Pioneering contributions to the development of neutron scattering techniques, for the studies of condensed matter.

1994-Clifford G. Shull, Born in USA (1915-2001) was awarded 1/2 of the Nobel Prize for the Development of the neutron diffraction technique.

1995-Martin L.Perl, Born in USA (1927) was awarded 1/2 of the Nobel Prize for the Discovery of the tau lepton.

1995-Frederick Reines, Born in USA (1918-1998) was awarded 1/2 of the Nobel Prize for the Detection of neutrino particle.

1996-David M. Lee, Born in USA (1931) was awarded 1/3 of the Nobel Prize for the Discovery of super fluidity in Helium-3.

1996-Douglas D.Osheroff, Born in USA (1945) was awarded 1/3 of the Nobel Prize for the Discovery of super fluidity in Helium-3.

1996-Robert C. Richardson, Born in USA (1937) was awarded 1/3 of the Nobel Prize for the Discovery of super fluidity in Helium-3.

1997-Steven Chu, Born in USA (1948) was awarded 1/3 of the Nobel Prize for the Development of methods to cool and trap atoms with laser light.

1997-Claude Cohen-Tannoudji, Born in Constantine, Algeria (1933) was awarded 1/3 of the Nobel Prize for the development of methods to cool and trap atoms with laser light.

1997-William D. Philips, Born in USA (1948) was awarded 1/3 of the Nobel Prize for the Development of methods to cool and trap atoms with laser light.

1998-Robert B. Laughlin, Born in USA (1950) was awarded 1/3 of the Nobel Prize for the discovery of a new form of quantum fluid with fractionally charged excitations.

1998-Horst L. Stormer, Born in Federal Republic of Germany (1949) was awarded 1/3 of the Nobel Prize for the Discovery of a new form of quantum fluid with fractionally charged excitations.

1998-Daniel C. Tsui, Born in Henan, China (1939) was awarded 1/3 of the Nobel Prize for the Discovery of a new form of quantum fluid with fractionally charged excitations.

1999-Gerardus t'Hooft, Born in the Netherlands (1946) was awarded 1/2 of the Nobel Prize for Elucidating the quantum structure of electro-weak interactions in Physics.

1999-Martinus J. G.Veltman, Born in Bilthoven, the Netherlands (1931), was awarded 1/2 of the Nobel Prize for Elucidating the quantum structure of electroweak interactions in Physics.

2000-Zhores I. Alferov, Born in Russia (1930) was awarded 1/4 of the Nobel Prize for the Basic work on information and communication technology, in particular for developing semiconductor hetero structure used in high- speed and Opto-electronics.

2000-Herbert Kroemer, Born in Federal Republic of Germany (1928) was awarded 1/4 of the Nobel Prize for the Basic work on information and communication technology, in particular for developing semiconductor hetero structure used in high speed and Opto-electronics.

2000-Jack S.Kilby, Born in USA (1923) was awarded 1/2 of the Nobel Prize for the Basic work on information and communication technology, in particular for the invention of integrated circuit.

2001-Eric A. Cornell, Born in USA (1961) was awarded 1/3 of the Nobel Prize for the achievement of BoseEinstein condensation in dilute gases of alkali atoms, and for early fundamental studies of the properties of the condensates.

2001- Wolfgang Ketterle, Born in Federal Republic of Germany (1957) was awarded 1/3 of the Nobel Prize for the achievement of Bose-Einstein condensation in dilute gases of alkali atoms, and for early fundamental studies of the properties of the condensates.

2001- Carl E. Wieman, Born in USA (1951) was awarded 1/3 of the Nobel Prize for the achievement of BoseEinstein condensation in dilute gases of alkali atoms, and for early fundamental studies of the properties of the condensates. 
2002-Riccardo Giacconi, Born in Genoa, Italy (1931) was awarded 1/2 of the Nobel Prize for the Pioneering contributions to astro-physics, which have led to the discovery of cosmic X-ray sources.

2002-Masatoshi Koshiba, Born in Japan (1926) was awarded 1/4 of the Nobel Prize for the Pioneering contributions to astro-physics, in particular for the detection of cosmic neutrinos (neutrino-astronomy).

2002-Raymond Davis Jr., Born in USA (1914) was awarded 1/4 of the Nobel Prize for the Pioneering contributions to Astro-Physics, in particular for the detection of cosmic neutrinos (neutrino-astronomy).

2003-Anthony Leggett, Born in United Kingdom (1938) was awarded 1/3 of the Nobel Prize for Pioneering contributions to the theory of superconductors and super fluidity.

2003-Alexei A.Abrikosov, Born in USA (1928) was awarded 1/3 of the Nobel Prize for Pioneering contributions to the theory of superconductors and super fluidity.

2003-Vitaly Ginzburg, Born in Russia (1916) was awarded 1/3 of the Nobel Prize for the contributions to the theory of superconductors and super fluidity.

2004-David J. Gross, Born in (1941) was awarded 1/3 of the Nobel Prize for the discovery of asymptotic freedom in the theory of the strong interaction.

2004-H. David Politzer, Born in (1949) was awarded 1/3 of the Nobel Prize for the Discovery of asymptotic freedom in the theory of the strong interaction.

2004-Frank Wilczek, Born (1951) was awarded 1/3 of the Nobel Prize for the discovery of asymptotic freedom in the theory of the strong interaction.

2005-Roy J. Glauber, was awarded one-half of the Nobel Prize for the contribution to the Quantum theory of optical coherence.

2005-John L. Hall, was awarded one-fourth of the Nobel Prize for the contributions to the development of laserbased precision spectroscopy, including the optical frequency comb technique.

2005-Theodore W. Hansch, was awarded one-fourth of the Nobel Prize for the contributions to the development of laser-based precision spectroscopy, including the optical frequency comb technique.

2006-John C. Mather, was awarded one-half of the Nobel Prize for the discovery of the blackbody form and anisotropy of the cosmic micro-wave background radiation.

2006-George F.Smoot, was awarded one-half of the Nobel Prize for the discovery of the blackbody form and anisotropy of the cosmic micro-wave background radiation.

2007-Albert Fert, was awarded one-half of the Nobel Prize for the discovery of Giant magneto resistance.

2007-Peter Grunberg, was awarded one-half of the Nobel Prize for the discovery of Giant magneto resistance.

2008-Yoichiro Nambu, was awarded one-half of the Nobel Prize for the discovery of the mechanism of spontaneous broken symmetry in sub- atomic physics.

2008-Makoto Kobayashi, was awarded one-fourth of the Nobel Prize for the discovery of the origin of the broken symmetry which predicts the existence of at least three families of quarks in nature.

2008-Toshihide Maskawa, was awarded one-fourth of the Nobel Prize for the discovery of the origin of the broken symmetry which predicts the existence of at least three families of quarks in nature.

2009-Charles Kuen Kao, was awarded one-half of the Nobel Prize for the ground breaking achievements concerning the transmission of light in fibers for optical communication.

2009-Willard S.Boyle,was awarded one-fourth of the Nobel Prize for the invention of an imaging semiconductor circuit- the CCD sensor.

2009-George E.Smith,was awarded one-fourth of the Nobel Prize for the invention of an imaging semiconductor circuit- the CCD sensor.

2010-Andre Geim, was awarded one-half of the Nobel Prize for the Ground breaking experiments regarding the two dimensional material graphene.

2010-Konstantin Novoselov, was awarded one-half of the Nobel Prize for the ground breaking experiments regarding the two dimensional material graphene.

2011-Saul Perlmutter was born in United States of America in 1959. He was awarded $1 / 2$ of the Nobel Prize for the discovery of the "Accelerating expansion of the Universe through the observations of distant supernovae".

2011-Brian Schmidt was born in Montana in USA in February 1967. He was awarded 1/4 of the Nobel Prize for the discovery of the"Accelerating expansion of universe through the observations of distant supernovae".

2011-Adam Riess was born in Washington D.C. in December 1969. He was awarded 1/4 of the Nobel Prize for the discovery of the "Accelerating expansion of the Universe through observations of distant supernovae".

2012-Serge Haroche was born in Casablanca, Morocco in 1944. He was awarded $1 / 2$ of the Nobel Prize in Physics "for ground-breaking experimental methods that enable measuring and manipulation of individual quantum systems".

2012-David J.Wineland was born in Milwaukee, WI, USA in 1944. He was awarded $1 / 2$ of the Nobel Prize in Physics "for ground-breaking experimental methods that enable measuring and manipulation of individual quantum systems".

2013- Peter Higgs was born on $29^{\text {th }}$ May 1929 in Newcastle Upon Tyne, United Kingdom. He is awarded $1 / 2$ of 
the Nobel Prize in Physics for "The theoretical discovery of the mechanism that contribute to our understanding of the origin of mass of subatomic particles, and which recently was confirmed through the discovery of the predicted fundamental particles, by the CMS and ATLAS Experiments at CERN's Large Hadron Collider".

2013- Francois Englert was born on $6^{\text {th }}$ November 1932 in Etterbeck, Belgium."The theoretical discovery of the mechanism that contribute to our understanding of the origin of mass of subatomic particles, and which recently was confirmed through the discovery of the predicted fundamental particles, by the CMS and ATLAS Experiments at CERN's Large Hadron Collider".

\section{Reference:}

[1]. College Physics By R.L.Webber, K.V.Manning, M.W.White and A.G.Weygand

[2]. Concepts of Modern Physics By Arthur Beiser.

[3]. http://www.nobel/laureates/physics. 